Zhangfei Su, J. Jay Leitch, Robert J. Faragher, Adrian L. Schwan, Jacek Lipkowski,

Gramicidin A ion channel formation in model phospholipid bilayers tethered to gold (111) electrode surfaces, Electrochim Acta, 2017,343,364-372

\title{
Gramicidin A ion channel formation in model phospholipid bilayers tethered to gold (111) electrode surfaces.
}

\author{
Zhangfei Su, J. Jay Leitch, Robert J. Faragher, Adrian L. Schwan, Jacek Lipkowski* \\ Department of Chemistry, University of Guelph, Guelph, Ontario, N1G 2W1, Canada \\ E-mail: jlipkows@uoguelph.ca \\ Fax: (519) 7661499 \\ Tel : (519) 8244120 ext 58543
}

\begin{abstract}
Electrochemical and polarization modulation infrared reflection absorption spectroscopy (PM-IRRAS) techniques were employed to study the ion channel properties of gramicidin A (gA) in tethered bilayer lipid membranes (tBLMs) with varying concentrations of $\mathrm{gA}$ added to the outer leaflet of the bilayer. The electrochemical impedance spectroscopy (EIS) results demonstrate that $\mathrm{gA}$ forms conductive ion channels in tBLM systems even at concentrations as low as $0.5 \mathrm{~mol} \%$. The impedance of the membrane depended on the $\mathrm{gA}$ concentration where a minimum was observed at $10 \mathrm{~mol} \%$. When the amount of $\mathrm{gA}$ in the bilayer is increased to a concentration above $10 \%$, the ion channels appear to arrange into a non-conducting state. PM-IRRAS spectra suggest that the gA molecules adopt a tilt angle of $\sim 29 \pm 4^{\circ}$ in the conductive ion channel state. In the non-conducting state, the average tilt angle of the gA molecules is $\sim 52 \pm 3^{\circ}$, which implies that gA peptides assume a random orientation in the tBLM.
\end{abstract}


Dedicated to Professor Masatoshi Osawa on the occasion of his $65^{\text {th }}$ birthday and in recognition of his outstanding contributions to electrochemistry

Keywords: gramicidin A, tethered bilayer lipid membrane, ion channel, EIS, electrochemistry, PM-IRRAS

\section{Introduction}

Tethered bilayer lipid membranes (tBLMs) are built on gold or mercury electrodes surfaces by self-assembling a monolayer (SAM) of thiolipids, consisting of hydrophobic hydrocarbon chains and a hydrophilic spacer terminated with a mercapto group [1, 2]. The outer leaflets of the tBLMs are formed by fusion of unilamellar phospholipid vesicles onto the thiolipid SAM. The hydrophilic spacer provides a water rich region between the electrode surface and the hydrophobic region of the bilayer. Therefore, tBLMs are promising tools for the research of the membrane-active peptides in a quasi-natural environment [3-5]. Many peptides, such as valinomycin [6, 7], gramicidin [7-12], alamethicin [13] and melittin $[8,9,14]$ were studied in tBLMs using electrochemical and surface sensitive methods, including SPR [6, 8], AFM [7, 8] and IR [11].

In this work, tBLMs are used to study the conductivity, conformation and orientation of gramicidin A ( $\mathrm{gA})$ ion channels. Gramicidin A is a linear peptide with the following primary structure:

formyl-L-Val-Gly-L-Ala-D-Leu-L-Ala-D-Val-L-Val-D-Val-L-Trp-D-Leu-L-Trp-D-Leu-L -Trp-D-Leu-L-Trp-ethanolamine.

The sequence of alternating L,D-amino acids allows gA to fold as a right handed $\beta$-helix stabilized by a $\beta$-sheet hydrogen bonding pattern. The peptide structure displays polymorphism leading to different conformations, which are denoted as $\beta^{m}$ where $m$ is the total number of residues per turn [15-17]. In lipid bilayers, the polar peptide groups form the interior lining of the helix providing a water lumen while the hydrophobic side chains are projecting outward toward the lipid matrix. The hydrogen bonds associated with the 
indole rings of the tryptophan side chains and the phospholipid polar head groups orient the gA helix such that the C-terminus is directed towards the membrane surface and the $\mathrm{N}$-terminus aligned with the hydrophobic interior. In the $\beta^{6.3}$ conformation, gramicidin peptides form dimers, which span the membrane. The dimer is stabilized by six intramolecular hydrogen bonds between the two N-terminus of the gA peptide in one leaflet with the N-terminus of the peptide in the opposing leaflet of the bilayer. It is now well accepted that this conformation corresponds to formation of ion channels allowing the translocation of monovalent cations across the lipid bilayer [18, 19]. Another conformation of the gramicidin in lipid bilayers is the double-stranded conformation, $\beta^{5.6}$, which does not display any ion channel properties [20].

EIS is an ideal tool to study the conductivity of ion channel and ionophore peptides in tBLMs. He et al. employed EIS to study the kinetics of gramicidin in the tBLM composed of mixed thiols [8]. Becucci et al. [10] found that the gramicidin dimer formation was increased by a single-file $\mathrm{K}^{+}$flux along the dimeric channels in a tBLM on a mercury surface. Steinem et al. [21] performed EIS measurements to study the conducting properties of gramicidin A in a floating bilayer membrane (fBLM) system, while Naumowicz and Figaszwski [22] performed EIS experiments on a membrane formed on Teflon. Kozuch et al. [11] combined EIS and surface enhanced IR absorption spectroscopy (SEIRAS) to study gramicidin in tBLMs on nanostructured gold surface. They observed a correlation between the intensity of the gA amide I band and the impedance of the tBLMs.

The photon polarization modulation infrared reflection absorption IR spectroscopy (PM-IRRAS) is a powerful technique that provides molecular level information about the membrane-active peptides in biomimetic membranes supported on metal electrode surfaces [23]. The IR spectra recorded using PM-IRRAS are characterized by a high S/N and are nearly insensitive to atmospheric $\mathrm{H}_{2} \mathrm{O}$ and $\mathrm{CO}_{2}$. The amide I band $(1610-1700$ $\mathrm{cm}^{-1}$ ) of peptides does not overlap with IR bands of the phospholipid matrix, therefore 
PM-IRRAS spectra can provide unique information concerning the conformation and orientation of peptides in the biomimetic membrane [23, 24]. Ha et al. applied PM-IRRAS to study gramicidin incorporated into a gold supported hybrid hexadecanthiol/DMPC bilayer transferred from the electrolyte to air, and found that gramicidin formed active ion channels in the hybrid bilayer [25]. Laredo et al. [26] performed in situ PM-IRRAS measurements of potential induced orientational and conformational changes of gramicidin D in a DMPC bilayer supported at the gold (111) electrode surface. In that study, the bilayer was adsorbed directly onto the surface of the gold electrode surface applying stress to the $\beta^{6.3}$ helix. The objective of the present work is to perform PM-IRRAS studies on gramicidin incorporated into tethered bilayers where the inner (cytosolic) leaflet is comprised of a self-assembled 2,3-di-O-phytanyl-sn-glycerol-1-tetraethylene glycol-D,L- $\alpha$-lipoic acid ester (DPTL) thiolipid monolayer (originally designed by Schiller et al. [2]) and the outer (extracellular) leaflet is composed of 1,2-di-O-phytanyl-sn-glycero-3-phosphocholine (DPhPC). Schematic structures of these two molecules are shown in Figure 1a. This design should eliminate the stress on the $\beta^{6.3}$ helix from the biomimetic system since the tetraethylene glycol (TEG) spacer will provide adequate separation between the hydrophobic region of bilayer and the gold surface. Although, several studies involving gramicidin biomimetic membranes have previously been performed, this work presents new information about the conformation and orientation of the gramicidin in tBLM as a function of its bilayer concentration and the voltage applied to the electrode. This new data shows the correlation between the conductivity of the ion channel with respect to the orientation of the gramicidin peptides within the membrane. These results are relevant for the development of biosensors in addition to providing a general understanding about the nature of the open and closed ion channel states.

\section{Experiment}




\subsection{Materials}

DPhPC was purchased from Avanti Polar Lipids. Gramicidin A, potassium perchlorate (Bioultra, 99.5\%), and deuterium oxide $\left(\mathrm{D}_{2} \mathrm{O}, 99.99\right.$ atom \% D) were obtained from Sigma-Aldrich. All of the above chemicals were used as purchased without any additional purification. DPTL was synthesized at the University of Guelph as
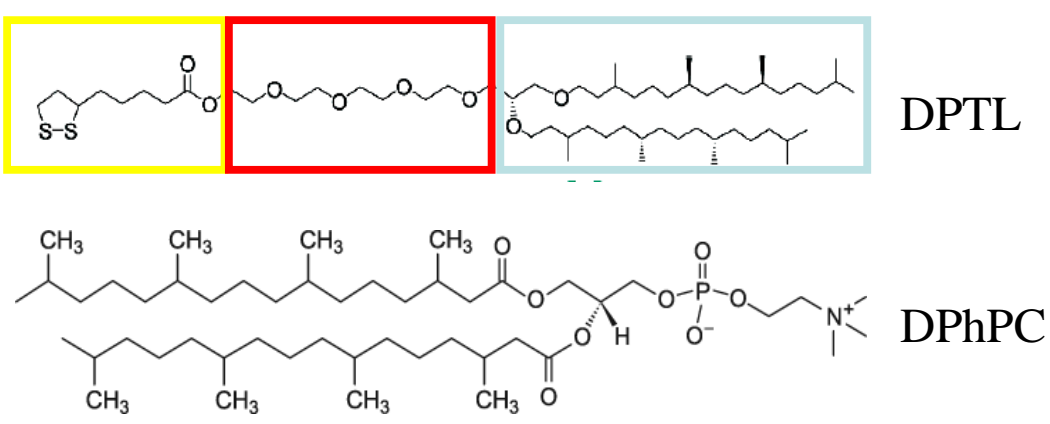

(b)

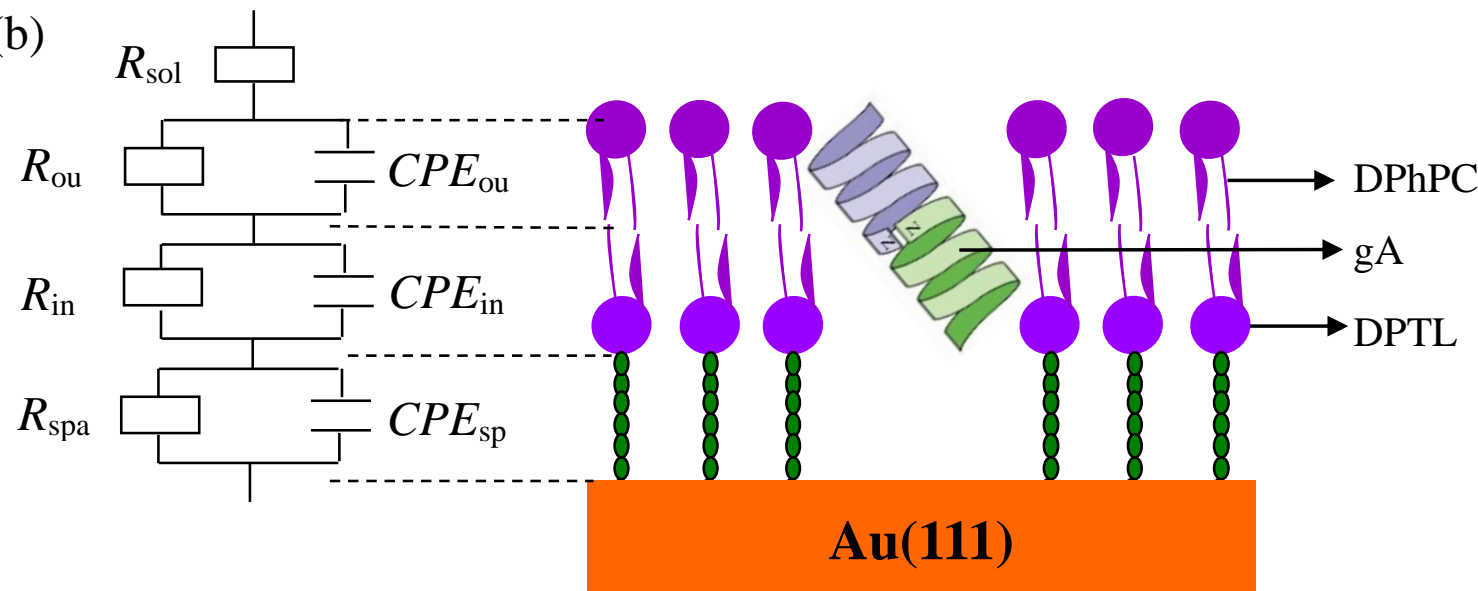

described in [27]. All aqueous solutions were prepared from ultrapure water purified by a Milli-Q UV plus (Millipore, Bedford, MA) water system (resistivity > $18.2 \mathrm{M} \Omega \mathrm{cm}$ ).

Figure 1. (a) Chemical structures of DPhPC and DPTL where the hydrophobic tails, hydrophilic space and mercapto anchor are highlighted with blue, red and yellow, respectively. (b) Schematic diagram of the gA-DPhPC (x:100-x) / DPTL bilayer at the Au (111) surface along with the equivalent circuit used in the EIS analysis.

The single crystal gold (111) electrodes, which were grown, oriented, and cut in our 
laboratory, were used as the working electrodes in the electrochemical and PM-IRRAS experiments. Prior to each experiment, the gold (111) electrode was flame-annealed and cooled in air. In the electrochemical experiments, a flame-annealed gold coil and $\mathrm{Ag} / \mathrm{AgCl}$ electrode (saturated $\mathrm{KCl}$ ), (Pine Research Instrumentation, $\mathrm{NC}$ ) were used as the counter and reference electrode, respectively $(+0.042 \mathrm{~V}$ versus the saturated calomel electrode (SCE)). In the PM-IRRAS measurements, a Pt foil and $\mathrm{Ag} / \mathrm{AgCl}$ (saturated $\mathrm{KCl}$ ) electrode were employed as the counter and reference electrode, respectively. All potentials reported in this paper are quoted versus the $\mathrm{Ag} / \mathrm{AgCl}$ (saturated $\mathrm{KCl}$ ) electrode. All experiments were performed at room temperature $\left(20 \pm 2^{\circ} \mathrm{C}\right)$.

\subsection{Construction of gA-DPhPC $(x: 100-x)$ / DPTL bilayer at the gold (111) surface}

The gA-DPhPC / DPTL bilayer with varying peptide to lipid (P/L) ratios was constructed on the gold (111) surface by the self-assembly of DPTL and subsequent fusion of gA-DPhPC vesicles. The gold (111) electrode was flame-annealed and then immersed into a $0.1 \mathrm{mg} \mathrm{mL}^{-1}$ methanolic (HPLC grade, Fisher Scientific) DPTL solution for a period of $12 \mathrm{~h}$ to form a uniform DPTL SAM on the gold (111) surface. Afterwards, the electrode was copiously rinsed with methanol and water, and then transferred to a 1 $\mathrm{mg} \mathrm{mL} \mathrm{m}^{-1} \mathrm{gA}-\mathrm{DPhPC}(x: 100-x)$ vesicle solution with different concentrations of $\mathrm{gA}(x)$ for another $12 \mathrm{~h}$ to create the outer bilayer leaflet on top of the self-assembled DPTL monolayer (inner leaflet) and ensure that the gA peptides were reconstituted into the tBLM. In this paper, $x$-refers to the mole fraction of gA delivered to the top leaflet. After completing the vesicle fusion, the tBLM-covered electrode was carefully rinsed with Milli-Q water.

The gA-DPhPC vesicle solution was prepared using the Barenholz method [28]. Stock solutions of gA and DPhPC in chloroform were individually added to a test tube to give a combined mass of $1 \mathrm{mg}$ for the peptides and the lipids. By adjusting the ratio between the volume of the peptide and lipid stock solutions, different P/L ratios in the 
mixture were achieved. The mixed solution was dried by vortexing in the test tube under a stream of argon. This procedure allowed for a thin film of the gA-DPhPC mixture to coat the inside wall of the test tube. The dried peptide-lipid film was placed in a vacuum desiccator for several hours, to remove residual solvent species. Finally, $1 \mathrm{~mL}$ of Milli-Q water was added into the test tube and the mixture was sonicated for 30 minutes to suspend mixed gA-DPhPC vesicles in solution.

\subsection{Electrochemical instrumentation and measurements}

Electrochemical measurements were carried out in an all-glass three-electrode cell. The cell was de-aerated by purging with argon for 30 minutes. To prevent the influx of oxygen, an argon blanket was maintained over the solution throughout the experiments. The surface of the $\mathrm{Au}(111)$ electrode was horizontally touched to the supporting electrolyte and then slowly raised to attain a hanging meniscus configuration where only the single crystal surface was in contact with the electrolyte solution.

Electrochemical impedance spectroscopy (EIS) was performed using the Solartron SI 187 electrochemical interface and Solartron SI 1260 impedance/gain-phase analyzer. EIS pectra were collected in the frequency range from $10^{-2}$ to $10^{4} \mathrm{~Hz}$ with a bias potential of $0.0 \mathrm{~V}$ and excitation amplitude of $0.01 \mathrm{~V}$. Data processing of the EIS results was conducted using the ZView software (Scribner Associates Inc.). The impedance spectra were fitted to an equivalent circuit as shown in Figure 1(b). $R_{\text {sol }}$ is the resistance of the electrolyte; $R_{\mathrm{ou}}$ and $C P E_{\mathrm{ou}}$ are the resistance and constant phase element of the outer leaflet of the bilayer; $R_{\mathrm{in}}$ and $C P E_{\mathrm{in}}, R_{\mathrm{sp}}$ and $C P E_{\mathrm{sp}}$ represent the resistances and constant phase elements of the inner and spacer layers, respectively. The constant phase element (CPE) was employed in the equivalent circuit to account for the heterogeneity of tBLMs. The impedance of the CPE can be expressed as:

$$
Z_{C P E}=\frac{1}{Q(j \omega)^{\alpha}}
$$


where $Q$ is the constant phase element coefficient measured in $\mu \mathrm{F} \mathrm{cm}{ }^{-2} \mathrm{~s}^{\alpha-1}$, and $\alpha$ is an empirical constant related to the frequency dispersion.

\subsection{PM-IRRAS experiment}

PM-IRRAS experiments were performed using a Thermo Nicolet Nexus 870 spectrometer (Madison, WI), equipped with an external tabletop optical mount (TOM) box, a MCT-A detector, a photoelastic modulator (Hinds Instruments PM-90 with a II/ZS50 ZnSe $50 \mathrm{kHz}$ optical head, Hillsboro, OR), and a demodulator (synchronous sampling demodulator, GWC Instruments, Madison, WI). The TOM box was purged throughout the experiment using $\mathrm{CO}_{2}$ and $\mathrm{H}_{2} \mathrm{O}$-free air, which was provided by a Whatman gas generator. The spectra were collected using in-house software, an Omnic macro, and a digital-to-analog converter (Omega, Stamford, CT) to control the potentiostat (EG\&GPAR362, Princeton, NJ). An electrochemical IR cell with a 1 inch $\mathrm{CaF}_{2}$ equilateral prism (BoXin, Changchun, China) was used in the IR experiment. Prior to each experiment, the window was washed with methanol and pure water, and then cleaned for 15 minutes in an UV ozone chamber. The electrolyte was de-aerated by purging with argon for approximately 30 minutes to remove oxygen. At each potential, 4000 IR scans were added and averaged using an instrumental resolution of $4 \mathrm{~cm}^{-1}$.

The PM-IRRAS spectra were measured with the PEM set for half-wave retardation at $1600 \mathrm{~cm}^{-1}$ for the $\mathrm{C}=\mathrm{O}$ and amide $\mathrm{I}$ band stretching region and $2900 \mathrm{~cm}^{-1}$ for the $\mathrm{C}-\mathrm{H}$ stretching region. The angle of incidence was set to $60^{\circ}$ to obtain a large enhancement of the mean square electric field strength (MSEFS) of the p-polarized photon on the gold surface. $\mathrm{D}_{2} \mathrm{O}$ electrolyte was used to avoid the intense IR absorption by $\mathrm{H}_{2} \mathrm{O}$ around 1650 and $3000 \mathrm{~cm}^{-1}$ region. The thickness of the thin layer cavity between the electrode surface and $\mathrm{CaF}_{2}$ prism was determined by comparing the experimental reflectivity spectrum of the thin layer cell, attenuated by the absorption by solvent molecules within the cavity, to the reflectivity spectrum calculated from the optical constants of the cell constituents as 
described in [29]. In the PM-IRRAS experiment, the measured signal, $\Delta S$, is proportional to the absorbance, $A$, of the molecules adsorbed on the electrode surface and defined as:

$$
\Delta S=\frac{2\left(I_{s}-I_{p}\right)}{I_{s}+I_{p}}=2.3 A=2.3 \Gamma \varepsilon
$$

where $I_{s}$ and $I_{p}$ are the intensities of the $s$ - and $p$-polarized radiation, $\Gamma$ is the surface concentration of the adsorbed species and $\varepsilon$ is the decimal molar absorption coefficient of the adsorbed species. The details of PM-IRRAS measurement and data processing are described in $[23,30]$.

\subsection{Simulation of the PM-IRRAS spectra}

The PM-IRRAS spectra of gA-DPhPC $(x: 100-x)$ / DPTL bilayer were simulated using a model of six homogeneous, parallel phases (Au/spacer/DPTL leaflet/gA-DPhPC leaflet $/ \mathrm{D}_{2} \mathrm{O} / \mathrm{CaF}_{2}$ ) and custom made software that solves the Fresnel equations employing the transfer matrix method [31]. The optical constants of gold, $\mathrm{D}_{2} \mathrm{O}$ and $\mathrm{CaF}_{2}$ were taken from literature [32-34]. The optical constants of the gramicidin-DPhPC mixture were measured from the transmission IR experiment of a dispersion of gramicidin-DPhPC vesicles in $\mathrm{D}_{2} \mathrm{O}$ using a thin-layer cell equipped with planar $\mathrm{CaF} 2$ windows. The optical constants of DPTL were taken from [35]. The optical constants of gA-DPhPC(10:90) mixture in the $\mathrm{C}=\mathrm{O}$ and amide $\mathrm{I}$ bands stretching region were plotted in Figure $\mathrm{S} 1$ of the Supplementary Information. The thickness of the inner leaflet corresponding to the hydrophobic portion of DPTL and the outer gA-DPhPC leaflet were assumed to be equal to half of the thickness of a DPhPC bilayer [36], and the thickness of the spacer between lipid bilayer and gold surface was assumed to be $\sim 3 \mathrm{~nm}$ based on the AFM and SPR data [7, 8]. Detailed information about the measurement of the optical constants and the simulation of PM-IRRAS spectra is described in [30].

\subsection{Calculating the tilt angles of the hydrocarbon lipid chains and $\mathrm{gA} \boldsymbol{\beta}^{6.3}$-helices}


When linearly polarized radiation is absorbed by species adsorbed at an electrode surface, the integrated intensity of the absorption band is proportional to the square of the dot product of the transition dipole vector $(\vec{\mu})$ and the electric field vector of the incident radiation $(\vec{E})$, shown as $[37,38]$ :

$$
\int A d v \propto \Gamma|\vec{\mu} \cdot \vec{E}|^{2}=\Gamma|\mu|^{2}|E|^{2} \cos ^{2} \theta
$$

At the metal electrode surface, the direction of the electric field vector of the $p$-polarized radiation is normal to the surface, hence the integrated absorption band intensity in the $p$-polarized spectra of the adsorbed species is proportional to $\cos ^{2} \theta$, where $\theta$ is the angle between the transition dipole of the vibration of the adsorbed species and the surface normal. For randomly oriented molecules, $\cos ^{2} \theta=1 / 3$. The orientation of adsorbed species can be determined from the ratio of the integrated intensity of a band in the measured spectrum and integrated intensity of this band in the simulated spectrum of randomly oriented molecules [37, 38]:

$$
\cos ^{2} \theta=\frac{\int A_{\exp } d v}{3 \int A_{c a l} d v}
$$

where $A_{\text {exp }}$ is the IR absorption band in the measured PM-IRRAS spectrum of adsorbed species and $A_{\text {cal }}$ is the absorption band in the simulated IR spectrum. The $A_{\text {cal }}$ can be simulated from the optical constants of adsorbed molecules as described in the preceding section. The error analysis in the determination of the angle $\theta$ was described in [26].

The direction of the transition dipole corresponding to the asymmetric stretch of the $\mathrm{CH}_{2}$ group is located along the line joining the two hydrogen atoms of the methylene group. For the $\mathrm{CH}_{2}$ symmetric stretching, the direction of the transition dipole lies along the bisector of the $\mathrm{CH}_{2}$ plane. The two vectors are orthogonal to each other and form a $90^{\circ}$ angle with the line of a fully extended all-trans hydrocarbon chain. Therefore, the angles $\theta_{\mathrm{as}}\left(\mathrm{CH}_{2}\right)$ and $\theta_{\mathrm{s}}\left(\mathrm{CH}_{2}\right)$ and the average tilt angle of trans fragments of the phytanyl chains $\left(\theta_{\text {chain }}\right)$ in DPhPC and DPTL are related by the equation [39]: 


$$
\cos ^{2} \theta_{a s}+\cos ^{2} \theta_{s}+\cos ^{2} \theta_{\text {chain }}=1
$$

The $\theta_{\mathrm{as}}\left(\mathrm{CH}_{2}\right)$ and $\theta_{\mathrm{s}}\left(\mathrm{CH}_{2}\right)$ can be calculated according to Eq. 4 from the integrated intensity of the $\mathrm{CH}_{2}$ asymmetric and symmetric stretching bands in the experimental and simulated spectra.

The average tilt angle of the $\beta^{6.3}$-helix of gA with respect to the surface normal can be calculated using the following steps [40]. First, one calculates the order parameter $\left(S_{\mathrm{am}}\right)$ for the amide I band [41]:

$$
S_{\mathrm{am}}=\frac{1}{2}\left(3 \cos ^{2} \theta_{\mathrm{am}}-1\right)
$$

$\theta_{\mathrm{am}}$ is the angle between the transition dipole of the amide I band and the surface normal calculated with the help of Eq.4. Next the $S_{\text {am }}$ is used to calculate order parameter of the $\beta^{6.3}$-helix $\left(S_{\text {helix }}\right)[41,42]$ :

$$
S_{\text {helix }}=\frac{2 S_{\mathrm{am}}}{3 \cos ^{2} \alpha-1}
$$

where $\alpha$ is the angle between the $\beta$-helix axis and the direction of the transition dipole of the amide I band. Finally, the average tilt angle of the $\beta$-helix axis (helix) can be determined from $S_{\text {helix }}$ using the formula:

$$
\gamma_{\text {helix }}=\arccos \sqrt{\frac{2 S_{\text {helix }}+1}{3}}
$$

Different values of $\alpha$ are reported in the literature. Kota et al. estimated the value of $\alpha$ to be $32^{\circ}$ from the polarized ATR spectroscopy experiment [43]. Ulrich et al. used a value of $10.8^{\circ}$ determined from the normal mode calculations for the $\beta^{6.3}$-helix $[44,45]$. Nabedryk et al. reported a value of $22.6^{\circ}$ for the $\beta^{4.4}$-helix based on the IR dichroism spectra of gramicidin in DMPC vesicles [46]. In the present paper, we assumed that $\alpha$ was equal to $32^{\circ}$, as previously reported by Kota et al [43], since this value has been used in our previous PM-IRRAS studies on gramicidin in the DMPC bilayer supported at the gold (111) surface [26]. 


\section{Results and Discussion}

\subsection{Electrochemical measurements}

In order to determine the potential drop across the tBLM, the potential of zero free charge $\left(E_{\mathrm{pzfc}}\right)$ has to be determined. The immersion method, described by Hamm et al. [47], was firstly applied by Ramirez et al [48] to determine $E_{\mathrm{pzfc}}$ of thiols monolayers covered gold electrode. Su et al. [49] and Seenath et al. [50] employed the immersion method to determine $E_{\mathrm{pzfc}}$ at the gold electrode covered with the assembled tBLM. In the present work, the gA-DPhPC (10:90) / DPTL tBLM covered electrode was dried in a stream of argon for about $30 \mathrm{~min}$ and then slowly brought in contact with the electrolyte at a constant electrode potential. The current flowing to the electrode when the contact is made was recorded. The inset to Figure 2 shows a representative transient that was measured. The procedure was repeated for a series of applied potentials. The current transients were integrated and the calculated charge densities were plotted versus the electrode potential in Figure2.

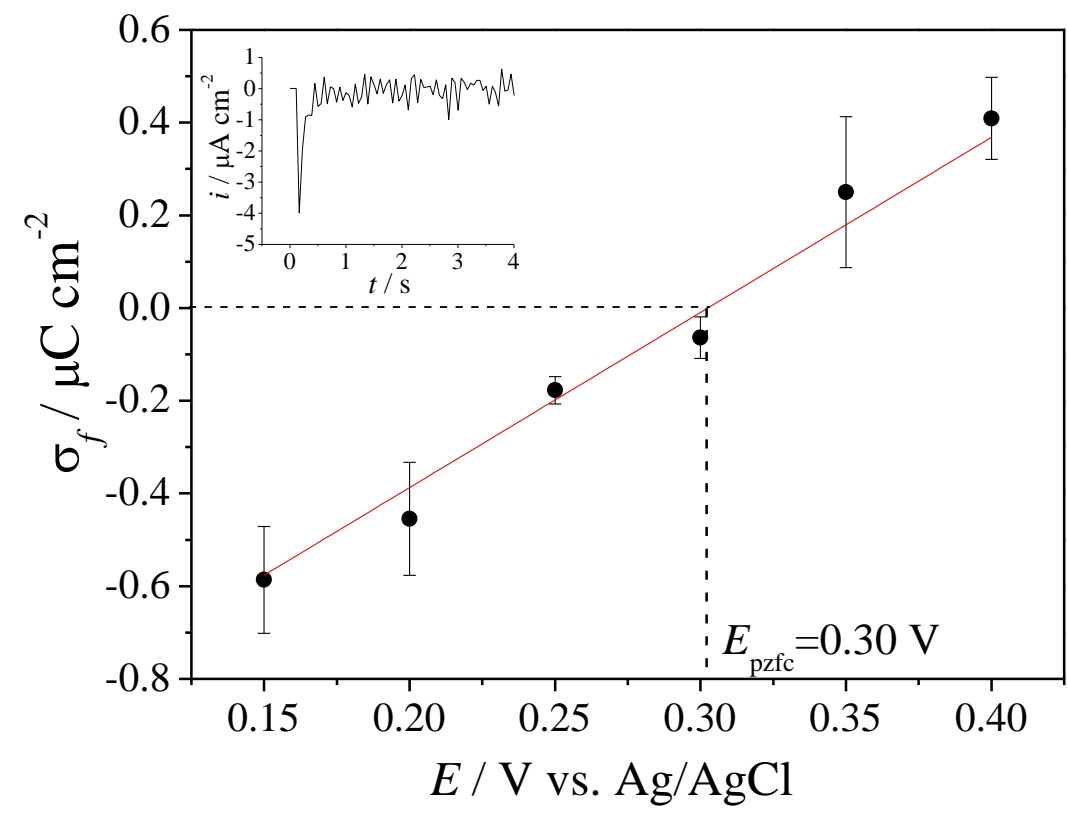

Figure 2. Charge densities determined by integration of the current flowing to the 
electrode when the gA-DPhPC(10:90) / DPTL tBLM covered Au(111) electrode was brought in contact with the $0.1 \mathrm{M} \mathrm{KClO}_{4}$ solution at the plotted applied electrode potentials. Inset shows representative current transient at $E=0.2 \mathrm{~V}$ vs $\mathrm{Ag} / \mathrm{AgCl}$ used to calculate the charge densities.

The linear regression of this data gives the value of the potential of zero free charge $E_{\mathrm{pzfc}}=0.30 \pm 0.03 \mathrm{~V}$ vs $\mathrm{Ag} / \mathrm{AgCl}$. This value is $\sim 0.07 \mathrm{~V}$ more positive that the potential of zero charge of the film free $\mathrm{Au}(111)$ electrode in $0.1 \mathrm{M} \mathrm{KClO}_{4}$ solution $(0.27 \mathrm{~V}$ vs SCE which corresponds to $0.23 \mathrm{~V}$ vs $\mathrm{Ag} / \mathrm{AgCl}[49])$. The $E_{\mathrm{pzfc}}$ of the tBLM is also $\sim 0.17 \mathrm{~V}$ more positive than the $E_{\mathrm{pzfc}}$ of the $\mathrm{Au}(111)$ electrode covered by the self-assembled monolayer of DPTL ( $0.17 \mathrm{~V}$ vs SCE or $0.13 \mathrm{~V}$ vs $\mathrm{Ag} / \mathrm{AgCl}$ [49]). The $E_{\text {pzfc }}$ is the potential at which the electrostatic charge at the tBLM covered electrode is equal to zero. Therefore the potential drop across the membrane due to charge on the metal is equal to:

$$
\Delta \phi_{M-2}=E-E_{\mathrm{pzfc}}-\phi_{2}
$$

where $\phi_{2}$ is the outer Helmholtz plane potential. At the electrode covered by tBLM the charge densities at the gold surface are small and hence $E$ - $E_{\mathrm{pzfc}}$ will be used as a measure of the potential drop across the membrane.

Figure 3 shows the cyclic voltammetry (CV) curves for the gold (111) electrode with tBLM containing different concentrations of $\mathrm{gA}$ in the extracellular leaflet. The shape of the voltammograms indicates that these experiments primarily measure the differential capacitance of the electrode. The CVs show that all tBLMs are stable on the gold (111) surface in the potential region between $-0.25 \mathrm{~V}$ to $+0.55 \mathrm{~V}$ vs $\mathrm{Ag} / \mathrm{AgCl}$ or transmembrane potentials -0.55 to $+0.25 \mathrm{~V}$. For the DPhPC/DPTL bilayer in the absence of $\mathrm{gA}$ (dashed line, $x=0$ ), the voltammetric current is small, demonstrating that this tBLM has a very low capacitance. After adding $0.5 \mathrm{~mol} \% \mathrm{gA}$ to the top leaflet of tBLM, the current increases, indicating that gramicidin causes an increase in the electrode capacitance. With increasing gramicidin concentration, the voltammetric current increases until a maximum value is achieved at 10 mol\% gramicidin delivered to the top leaflet. Further addition of 
gramicidin causes the decrease of the voltammetric current indicating a decrease of the capacitance. The CV curves demonstrate that gramicidin is inserted into the tBLMs, consistent with our previous studies of related systems $[26,51]$.

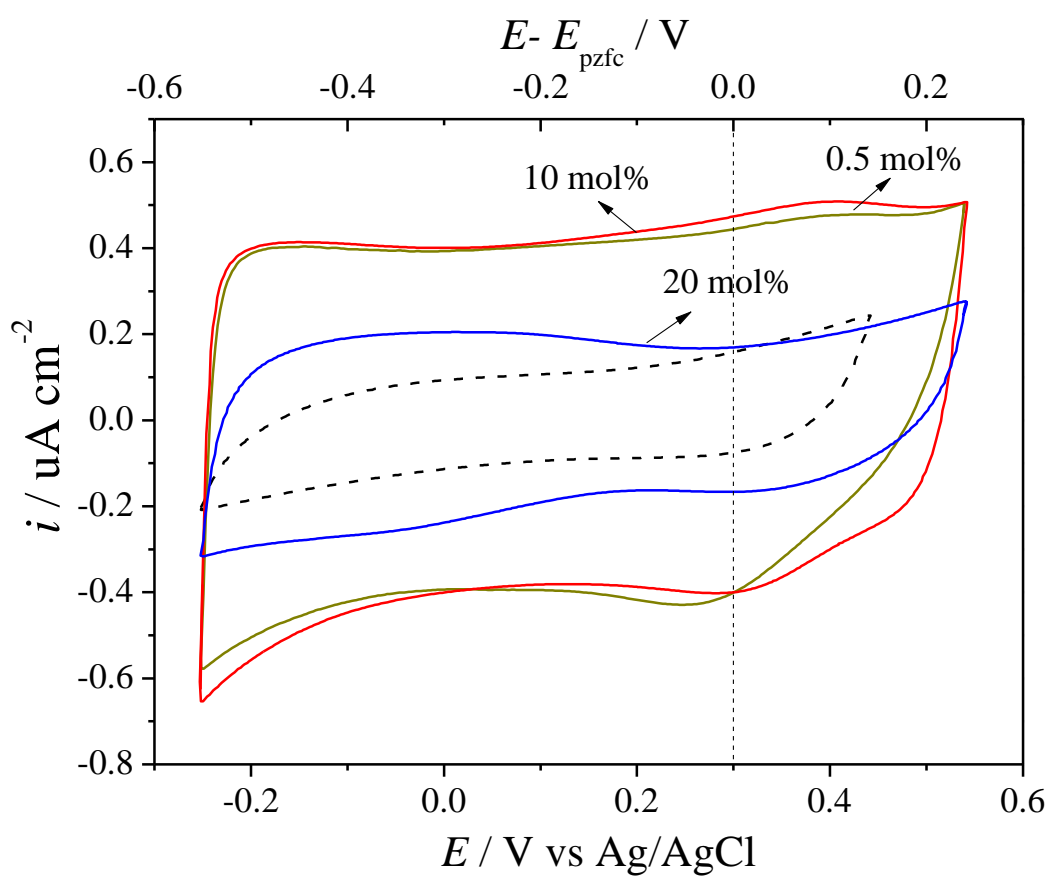

Figure 3. Cyclic voltammograms recorded at a scan rate of $20 \mathrm{mV} \mathrm{s}^{-1}$ for the gold (111) electrode with gA-DPhPC $(x: 100-x)$ / DPTL bilayers of different concentrations of gA $(x)$ delivered to the top leaflet; $\mathrm{CV}$ with the bilayer without gA: dashed line, in a 0.1 $\mathrm{M} \mathrm{KClO}_{4}$ electrolyte solution. Top horizontal axis plots the potential drop across the bilayer.

The CV curve for the tBLM with $10 \mathrm{~mol} \%$ gramicidin displays a pair of small peaks around $0.4 \mathrm{~V}$ and $0.3 \mathrm{~V}$. The separation between these peaks is $\sim 0.09 \mathrm{~V}$, which suggests a quasi-reversible process. The mid-separation potential is $\sim 0.35 \mathrm{~V}$ vs $\mathrm{Ag} / \mathrm{AgCl}$ and hence about $+0.05 \mathrm{~V}$ vs $E_{\text {pzfc. }}$ Becucci et al. [10] observed the presence of voltammetric peaks on CVs recorded at gramicidin-doped DPhPC/DPTL tethered bilayer assembled at the 
mercury electrode surface. These peaks were assigned to the transport of potassium ions across the membrane to and from the hydrophilic spacer layer. Incidentally, Becucci et al. $[52,53]$ estimated that the mid-separation potential between the peaks is observed at the potential difference across the lipid bilayer moiety of a tBLM approximately equal to zero. Therefore a similar behavior is observed for gramicidin molecules reconstituted into tBLM assembled at gold and mercury electrodes. However, the integrated area of the small peaks in Figure 3 is $0.58 \mu \mathrm{C} \mathrm{cm}^{-2}$. In contrast, Becucci et al. [10] reported the integrated charge density of $\mathrm{K}^{+}$peak to be $\sim 45 \mu \mathrm{C} \mathrm{cm}^{-2}$ corresponding to the maximum charge of $\mathrm{K}^{+}$ions that can be accommodated by the TEG spacer. The major difference between the charge density of $\mathrm{K}^{+}$ions in the present paper and that reported by Becucci et al. [10] may be due to the differences in the volume of water within the TEG spacer of the self-assembled tBLM on the gold and mercury electrodes. For DPTL on the gold (111) surface, the PM-IRRAS spectra revealed that a significant fraction of the TEG groups was not hydrated, indicating that the water content within the hydrophilic spacer region was small [35]. There is no information about the conformation of DPTL on the mercury electrode surface. However, the fluidity and lateral mobility of lipids in the tBLM on the mercury surface is higher than on the gold surface [54]. Therefore, the amount of water in the TEG spacer on the mercury surface may be larger than that on the gold (111) surface. Alternatively, gramicidin molecules may not reconstitute well into the bottom leaflet and form fewer channels in the tBLM assembled at the $\mathrm{Au}$ (111) surface. To address this issue, EIS measurements were performed.

The EIS measurements were carried out at the bias potential of $0.0 \mathrm{~V}$ vs. $\mathrm{Ag} / \mathrm{AgCl}$. Figure 4(a) plots the impedance of the $\mathrm{Au}$ (111) electrodes with tBLMs containing different concentrations of gramicidin. The impedance of the pure DPhPC/DPTL bilayer (black curve) is high. After the addition of $0.5 \mathrm{~mol} \%$ gramicidin into the top leaflet of the tBLM, the impedance greatly decreases. With increasing gramicidin concentration, the impedance of tBLMs further decreases until a minimum impedance value is reached at a 
gA concentration of $10 \mathrm{~mol} \%$. At this point, further additions of gramicidin to the tBLM
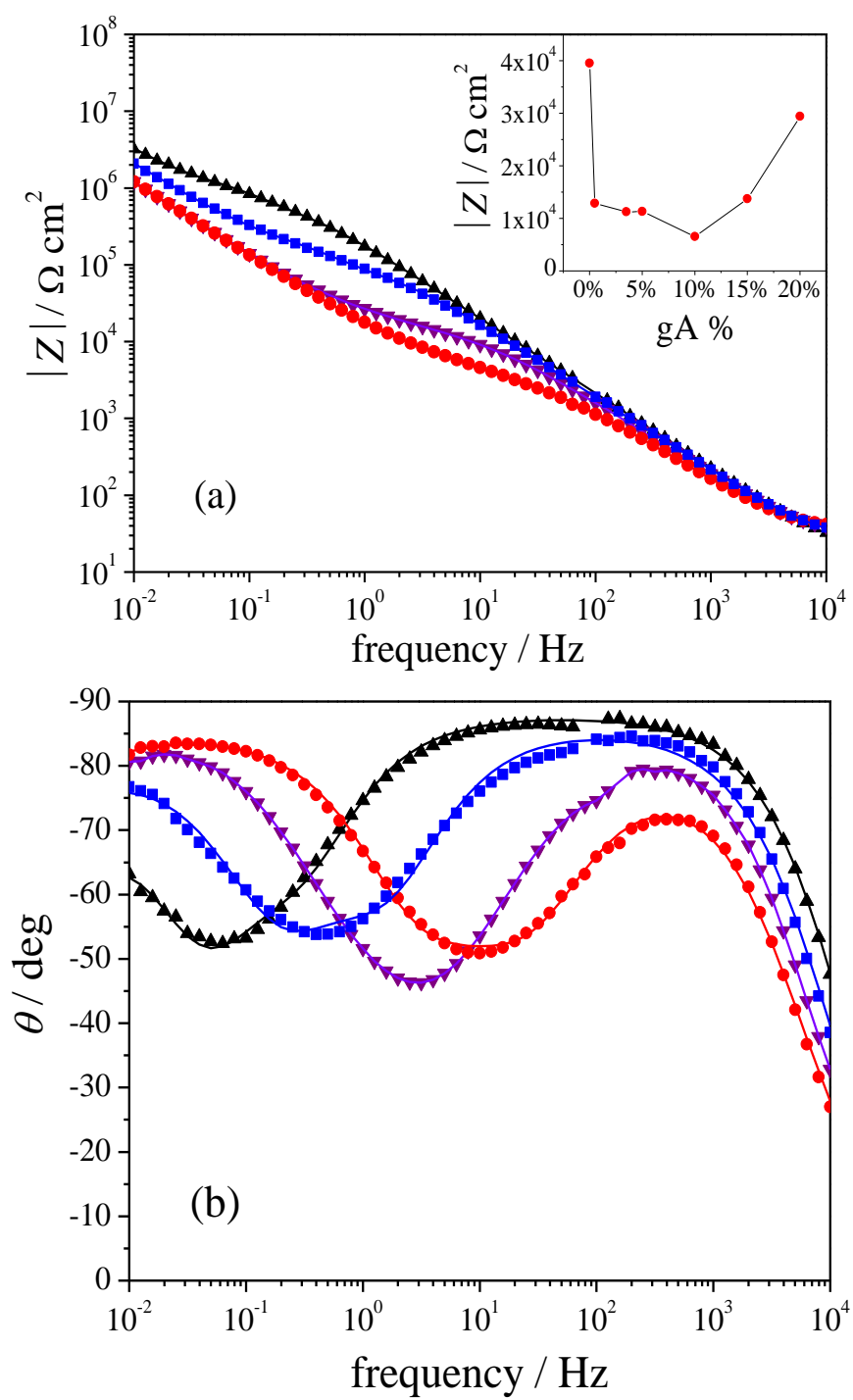

cause the total impedance to increase.

Figure 4. EIS spectra of gA-DPhPC $(x: 100-x)$ / DPTL bilayer at the gold (111) electrode in $0.1 \mathrm{M} \mathrm{KClO}_{4}$ at varying concentrations of gramicidin $(x)$. The pure DPhPC / DPTL bilayer is given by $x=0$ (black), $0.5 \mathrm{~mol} \% \mathrm{gA}$ (blue), $10 \mathrm{~mol} \% \mathrm{gA}(\mathrm{red})$ and $20 \mathrm{~mol} \% \mathrm{gA}$ (purple), $x$ refers to the mole fraction of gramicidin added to the top leaflet of the tBLM. The fitted curves were obtained via the equivalent circuit shown in Figure 1(b) where (a) plots the magnitude of the impedance and (b) presents the phase angle. The inset in Figure 3(a) presents the changes of the impedance as a function of the gA concentration 
within the tBLM at a fixed frequency of $5 \mathrm{~Hz}$.

Figure 4 (b) plots the corresponding changes of the phase angle. All curves display a minimum at the low frequency region $\left(f<10^{1} \mathrm{~Hz}\right)$. The presence of a minimum indicates that the equivalent circuit representing this data contains more than one time constant. Several equivalent circuits were tested and the simplest equivalent circuit that showed good agreement with the data set consisted of three meshes and resistance of the solution in series (see Figure 1b). Each mesh consists of a resistor and CPE element and a resistor. The CPE unit rather than a capacitance are used to allow for inhomogeneity of the Table 1 Numerical values of the elements of the equivalent circuit of $\mathrm{Au}(111)$ electrode with tethered bilayers gA-DPhPC $(x: 100-x)$ / DPTL in $0.1 \mathrm{M} \mathrm{KClO}_{4}$ and different concentrations of gramicidin $\mathrm{A}(x)$.

\begin{tabular}{|c|c|c|c|c|c|c|c|}
\hline \multicolumn{1}{|c|}{$x$} & 0 & 0.5 & 3.5 & 5 & 10 & 15 & 20 \\
\hline$Q$ of $\mathrm{CPE}_{\text {ou }} /$ & $3.02 \pm$ & $9.41 \pm$ & $10.0 \pm$ & $8.41 \pm$ & $11.8 \pm$ & $6.59 \pm$ & $5.27 \pm$ \\
$\mu \mathrm{F} \mathrm{cm}^{-2} \mathrm{~s}^{\alpha-1}$ & 0.62 & 0.74 & 0.6 & 1.0 & 0.6 & 0.22 & 0.93 \\
\hline$\alpha$ of $\mathrm{CPE}_{\text {ou }}$ & $0.981 \pm$ & $0.923 \pm$ & $0.957 \pm$ & $0.926 \pm$ & $0.879 \pm$ & $0.909 \pm$ & $0.963 \pm$ \\
& 0.10 & 0.06 & 0.018 & 0.064 & 0.050 & 0.037 & 0.094 \\
\hline$R_{\text {ou }} / \mathrm{k} \Omega \mathrm{cm}^{2}$ & $660 \pm$ & $11.0 \pm$ & $7.79 \pm$ & $7.30 \pm$ & $4.14 \pm$ & $26.6 \pm$ & $95.7 \pm$ \\
\hline$Q$ of $\mathrm{CPE}_{\text {in }} /$ & $1.66 \pm$ & $2.11 \pm$ & $2.46 \pm$ & $1.71 \pm$ & $3.58 \pm$ & $2.60 \pm$ & $1.89 \pm$ \\
$\mu \mathrm{F} \mathrm{cm} \mathrm{s}^{\alpha-1}$ & 0.35 & 0.05 & 0.04 & 0.04 & 0.01 & 0.10 & 0.15 \\
\hline$\alpha$ of $\mathrm{CPE}_{\text {in }}$ & $0.973 \pm$ & $0.941 \pm$ & $0.923 \pm$ & $0.954 \pm$ & $0.913 \pm$ & $0.949 \pm$ & $0.967 \pm$ \\
\hline$R_{\mathrm{i}} /{\mathrm{k} \Omega \mathrm{cm}^{2}}^{0.03}$ & 0.016 & 0.002 & 0.017 & 0.022 & 0.023 & 0.026 \\
\hline$Q$ of $\mathrm{CPE}_{\mathrm{sp}} /$ & $4.86 \pm$ & $11.2 \pm$ & $11.0 \pm$ & $12.3 \pm$ & $11.5 \pm$ & $11.7 \pm$ & $6.00 \pm$ \\
$\mu \mathrm{F} \mathrm{cm} \mathrm{s}^{\alpha-1}$ & 0.87 & 0.1 & 0.1 & 0.1 & 0.1 & 0.1 & 0.24 \\
\hline$\alpha$ of $\mathrm{CPE}_{\mathrm{sp}}$ & $0.968 \pm$ & $0.933 \pm$ & $0.942 \pm$ & $0.947 \pm$ & $0.944 \pm$ & $0.913 \pm$ & $0.911 \pm$ \\
\hline
\end{tabular}




\begin{tabular}{|l|c|c|c|c|c|c|c|}
\hline & 0.056 & 0.004 & 0.002 & 0.002 & 0.002 & 0.005 & 0.018 \\
\hline$R_{\mathrm{sp}} / \mathrm{M} \Omega \mathrm{cm}^{2}$ & $17 \pm 5$ & $35 \pm 13$ & $33 \pm 12$ & $33 \pm 8$ & $27 \pm 6$ & $36 \pm 9$ & $44 \pm 14$ \\
\hline
\end{tabular}

membrane. The solid lines in Figure 4 denote the fits of the equivalent circuit to the measured values. The calculated EIS curves show good fits to the experimental data.
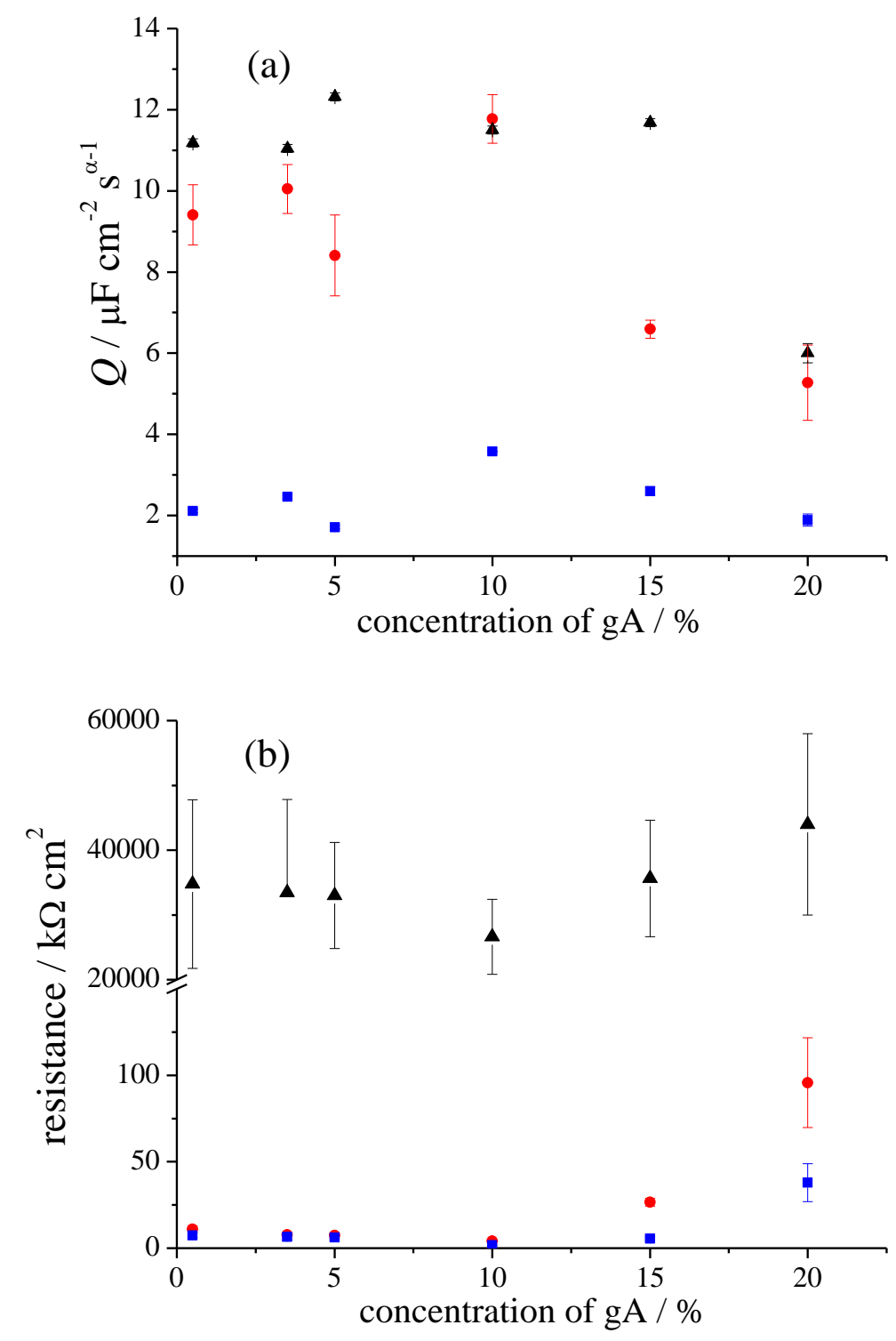

Figure 5. Dependence of the constant phase element $Q$ [Panel (a)] and resistance $R$ [Panel (b)] of the three meshes of the equivalent circuit shown in Figure 1b (red circles outer leaflet; blue squares - inner leaflet; black triangles - TEG spacer layer) on the concentration of gA in the gA-DPhPC $(x: 100-x)$ / DPTL bilayer. 
The numerical values of the elements of equivalent circuit determined from the fits are listed in Table 1. In addition, Figure 5 plots the dependence of the equivalent circuit elements on the concentration of gA added to the top leaflet of the tBLMs. The $Q$ of the $\mathrm{CPE}$ element is a measure of the capacitance for each mesh region of the bilayer. Figure $1 \mathrm{~b}$ shows that the three meshes of parallel CPEs and resistances are assigned to the outer and inner leaflets and the spacer layer. For a similar bilayer assembled at a $\mathrm{Hg}$ electrode, Becucci et al. [10] used similar equivalent circuit. However, they assigned the three meshes to the lipoic acid residue, the hydrophilic spacer, and the lipid bilayer moiety respectively. The difference between the present assignment and that in ref [10] illustrates that the assignment is not unique and subject to individual preferences. In the present study, the similarity between the values of parameters obtained for the two meshes assigned to the outer and inner leaflets of the bilayer, justify the assignment proposed in Figure $1 b$.

The data in Table 1 and Figure 5(a) show that the capacitance of the outer leaflet is always larger than the capacitance of the inner leaflet. In addition, the coefficient $\alpha$ is frequently somewhat smaller for the outer layer compared to the inner layer indicating that the outer leaflet is somewhat less homogeneous. The outer leaflet is formed by vesicle fusion and remains in contact with the solution. Hence it may contain more defects allowing more water molecules to penetrate this leaflet. The capacitance of the spacer layer is higher than of the two leaflets. This is reasonable since the spacer layer contains polar ethylene groups. The resistance of the spacer layer is very high in the range of $\mathrm{M} \Omega \mathrm{cm}^{2}$ and their values have high uncertainties. We have made attempts to model this region using only capacitance; however the fit was not satisfactory. The high resistance of the spacer region is consistent with the low water content in this layer as shown by Leitch et al. [35] and very small penetration of potassium ions demonstrated by the shape of CVs. The data in Table 1 and Figure 5(b) show that the addition of gA to 
the DPhPC/DPTL bilayer results in both an increase in the capacitance of all elements within the equivalent circuit, as previously observed earlier in the CV curves of Figure 3, as well as a decrease in the resistance of the resistive elements of the two leaflets of the bilayer. More significantly, the decrease in resistance takes place for both leaflets and the resistances of the two leaflets are comparable in magnitude. This indicates that gA must also be reconstituted into the inner leaflet of the bilayer. This is important result since the presence of $\mathrm{gA}$ in the two leaflets is required for the formation of the $\mathrm{gA}$ channels. The resistance attains a minimum at $10 \mathrm{~mol} \%$ of $\mathrm{gA}$ added to the top leaflet. A further increase in the $\mathrm{gA}$ concentration leads to an overall increase in all three resistive elements of the equivalent circuit. Below we employ PM-IRRAS spectroscopy to explain these behaviors.

\subsection{PM-IRRAS spectroscopy}

The electrochemical measurements indicated that gramicidin reconstituted into the tBLM has ion channel properties when the concentration added to the top leaflet is smaller than $10 \mathrm{~mol} \%$ and the formation of ion channels in the tBLM is reduced at high gA concentrations. The PM-IRRAS technique was applied to obtain information about the conformation and orientation of $\mathrm{gA}$ in the tBLMs and provide molecular level interpretation to the electrochemical data. Figure 6a plots the normalized PM-IRRAS spectra for the $\mathrm{C}=\mathrm{O}$ and amide I stretching bands of three bilayers, $0 \mathrm{~mol} \%, 10 \mathrm{~mol} \%$ and $20 \mathrm{~mol} \%$ of $\mathrm{gA}$ added to the top leaflet, recorded at an electrode potential of $0.0 \mathrm{~V}$ vs $\mathrm{Ag} / \mathrm{AgCl}$.

Since the spectra were recorded in independent experiments, the surface enhancement of the electric field of the photon at the electrode surface $\left\langle E_{\mathrm{p}, \mathrm{z}=0}^{2}\right\rangle \mid\left\langle E_{\mathrm{i}}^{2}\right\rangle$ had to be normalized to take into account small changes of the angle of incidence and 

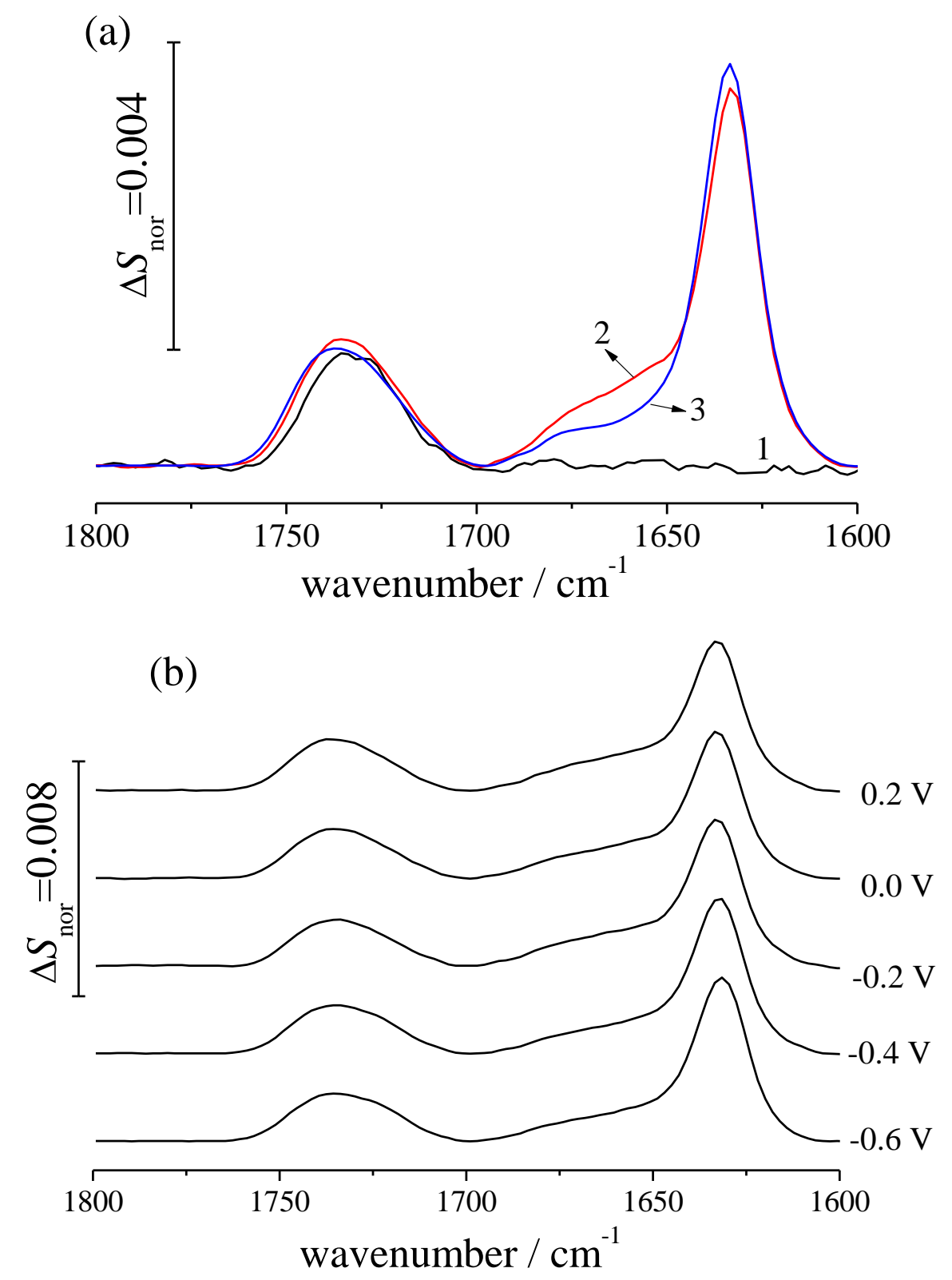

Figure 6. (a) Normalized PM-IRRAS spectra of DPhPC/DPTL (1, black), gA-DPhPC(10:90) / DPTL (2, red) and gA-DPhPC(20:80) / DPTL (3, blue) bilayers at the gold (111) electrode surface in the $\mathrm{C}=\mathrm{O}$ stretching and amide $\mathrm{I}$ regions in $0.1 \mathrm{M}$ $\mathrm{KClO}_{4} / \mathrm{D}_{2} \mathrm{O}$, and $E=0.0 \mathrm{~V}$ versus $\mathrm{Ag} / \mathrm{AgCl}$. (b) Normalized PM-IRRAS spectra of gA-DPhPC(10:90) / DPTL bilayer recorded at different electrode potentials vs. Ag/AgCl.

thickness of the gap between the electrode surface and the IR window. The normalized IR spectra $\left(\Delta S_{\text {nor }}\right)$ are defined as: 


$$
\Delta S_{\text {nor }}=\frac{\Delta S}{\left.<E_{p, z=0}^{2}>/<E_{i}^{2}\right\rangle}
$$

where $\Delta S$ is the experimental PM-IRRAS signal, and $\left\langle E_{p, z=0}^{2}\right\rangle$ is the mean square electric field strength (MSEFS) of the $p$-polarized beam at gold (111) electrode surface and $\left\langle E^{2}{ }_{\mathrm{i}}\right\rangle$ is the MSEFS of the incident $p$-polarized beam. The surface enhancement was calculated by solving Fresnel equations for a model of six stratified layers (Au/spacer/DPTL-leaflet/gA-DPhPC-leaflet/ $\mathrm{D}_{2} \mathrm{O} \mathrm{CaF}_{2}$ ) [30]. The normalization of the spectra eliminates the changes of the optical setup.

The band at $\sim 1732 \mathrm{~cm}^{-1}$, corresponds to the $\mathrm{C}=\mathrm{O}$ stretching in DPhPC and DPTL molecules. No IR bands are observed in the 1600 and $1700 \mathrm{~cm}^{-1}$ region for the IR spectrum of DPhPC/DPTL without gA where the amide I band of gA would be expected, while the spectra for tBLMs with 10 and $20 \mathrm{~mol} \%$ of the peptide added to the top leaflet both show a very distinctive amide I band suggesting that gA has incorporated into the bilayer. Figure 6(b) shows the PM-IRRAS spectra recorded at different electrode potentials versus $\mathrm{Ag} / \mathrm{AgCl}$ for the bilayer with $10 \mathrm{~mol} \%$ of $\mathrm{gA}$. The spectra show a small change with the electrode potential. The amide I band is broad and consists of several sub-bands. The generalized two-dimensional correlation spectroscopy (2D-COS) [55] and Fourier self-deconvolution (FSD) [56] were employed to identify the overlapping sub-bands in this region. The 2D-COS and FSD analyses are presented in Figures S2 and $\mathrm{S} 3$ of the Supporting Information. For the tBLM with $10 \mathrm{~mol} \%$ of gA added to the top leaflet, the band deconvolution is shown in Figure 7. The strong band at $\sim 1632 \mathrm{~cm}^{-1}$ corresponds to the $\beta^{6.3}$-helix that forms the ion conducting dimmer (HD) while bands at $1625,1644,1653,1660,1669,1678 \mathrm{~cm}^{-1}$ can be assigned to the $\beta^{5.6}$-double helix (DH) conformation [44]. The relative area of the $1632 \mathrm{~cm}^{-1}$ band is $\sim 60 \%$ in demonstrating that the $\beta^{6.3}$-helix structure is the predominant secondary structure of gA in tBLMs. This result is in agreement with the previous PM-IRRAS studies of gramicidin in the gold (111) supported DMPC bilayer [26]. 


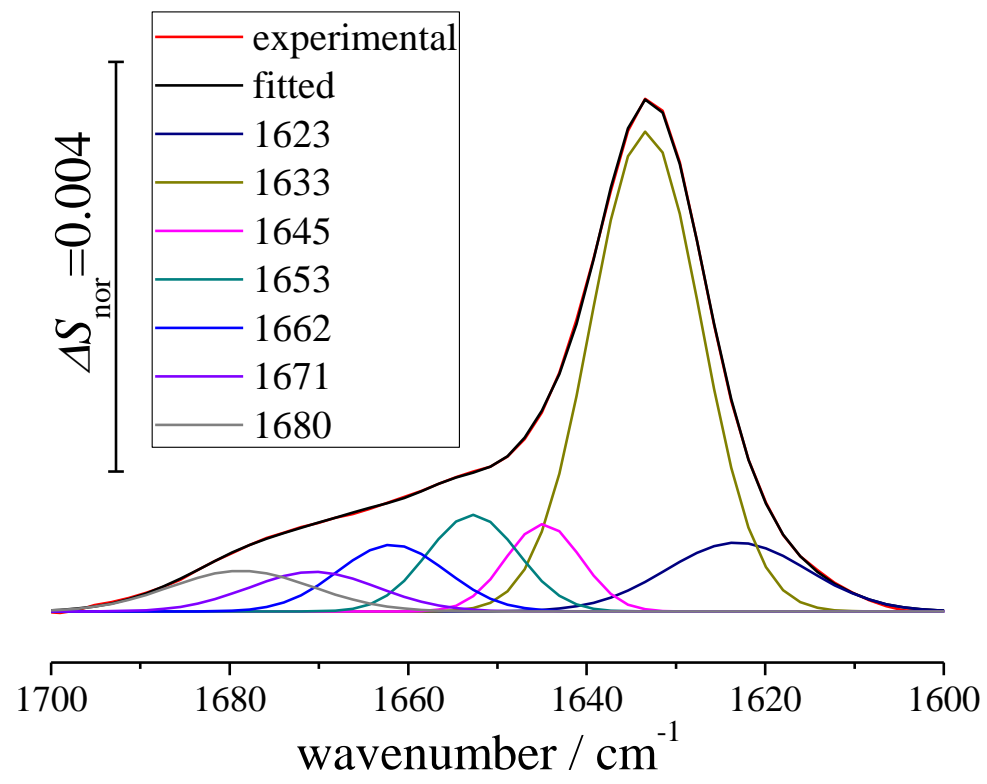

Figure 7. Deconvolution results of the amide I band of gramicidin in the gA-DPhPC (10:90) / DPTL bilayer.

The intensity of the amide I band depends on the concentration and orientation of gA molecules. The direction of the transition dipole with respect to surface normal for the $\sim 1632 \mathrm{~cm}^{-1}$ band can be determined with the help of Eq. 4 . The average tilt angle of the $\beta^{6.3}$-helix axis ( helix ) of gA can then be calculated using Eqs. 6, 7 and 8. The calculated angles are plotted as a function of the applied potential in Figure 8(a). For comparison the data reported previously for gramicidin in DMPC bilayers [26] are also included in this figure. For potentials more positive than $-0.4 \mathrm{~V}$, the tilt of the $\beta^{6.3}$-helix axis is $29 \pm 4^{\circ}$ in the gA-DPhPC(10:90) / DPTL bilayer in good agreement with the value of $25 \pm 2^{\circ}$ previously reported for gramicidin in DMPC bilayers [26]. The tilt angle of $29 \pm 4^{\circ}$ is small and allows gA molecules in the two leaflets to form the ion channel. This result is consistent with the EIS data that demonstrated a low resistivity of the bilayer with 10 mol\% of the gramicidin. Therefore, the low resistivity can be explained by formation of ion channels that allow for the transport of potassium ion across the membrane. In the gA-DPhPC(10:90) / DPTL bilayer the tilt angle decreases to $\sim 22^{\circ}$, at potentials more 
negative than -0.4 V. A similar trend was observed for gramicidin in DMPC bilayers. This change is caused by progressive dewetting of the bilayers from the gold electrode surface and penetration of water in the region between the membrane and the gold surface [26]. For both gA-DPhPC(20:80) / DPTL bilayer and the DMPC bilayer, the onset of dewetting is observed at transmembrane potentials - $0.4 \mathrm{~V}$. In the gA-DPhPC(20:80) / DPTL bilayer, thelix is equal to $52 \pm 3^{\circ}$, which indicates that the helices of gramicidin molecules are oriented randomly in the tBLM (Note: $55^{\circ}$ is the tilt of randomly oriented molecules). This orientation prevents the ion channel formation and explains why the EIS data shows an increase in membrane resistivity with $20 \mathrm{~mol} \%$ of the gramicidin.

Figure 8(b) plots the peak center of the $\beta^{6.3}$-helix IR band in the gA-DPhPC(10:90) / DPTL and gA-DPhPC(20:80) / DPTL tethered bilayers as a function of the electrode potential. For comparison, the data for gramicidin reconstituted into the DMPC bilayer supported at the $\mathrm{Au}(111)$ surface (taken from Ref. [26]) are also included in this figure. The amide I band corresponds predominantly to the collective vibrations of the $\mathrm{C}=\mathrm{O}$ stretching of the amide bond. The frequency of the amide I band depends on the transition dipole coupling mechanism (TDC) [57] and the strength of intramolecular hydrogen bonds. TDC originates from resonance interaction between the oscillating dipoles of neighboring amide groups. The strength of TDC depends on the relative orientation and the distance between the dipoles. The lower wavenumber of the peak center of the amide I band indicates stronger TDC and/or stronger intramolecular hydrogen bonds. This is the fundamental mechanism that causes the amide I band to be sensitive to the secondary structure of peptides. Figure $8(\mathrm{~b})$ shows that the peak center of the $\beta^{6.3}$-helix in tBLM with $10 \mathrm{~mol} \%$ of $\mathrm{gA}$ is located at lower wavenumbers than for gramicidin in the bilayer of DMPC supported at the Au(111) surface. This indicates stronger TDC for gramicidin in the tBLM than in the supported bilayer. As expected, the separation of the membrane from the metal surface by the TEG spacer released the stress in the $\beta^{6.3}$-helix induced by a 

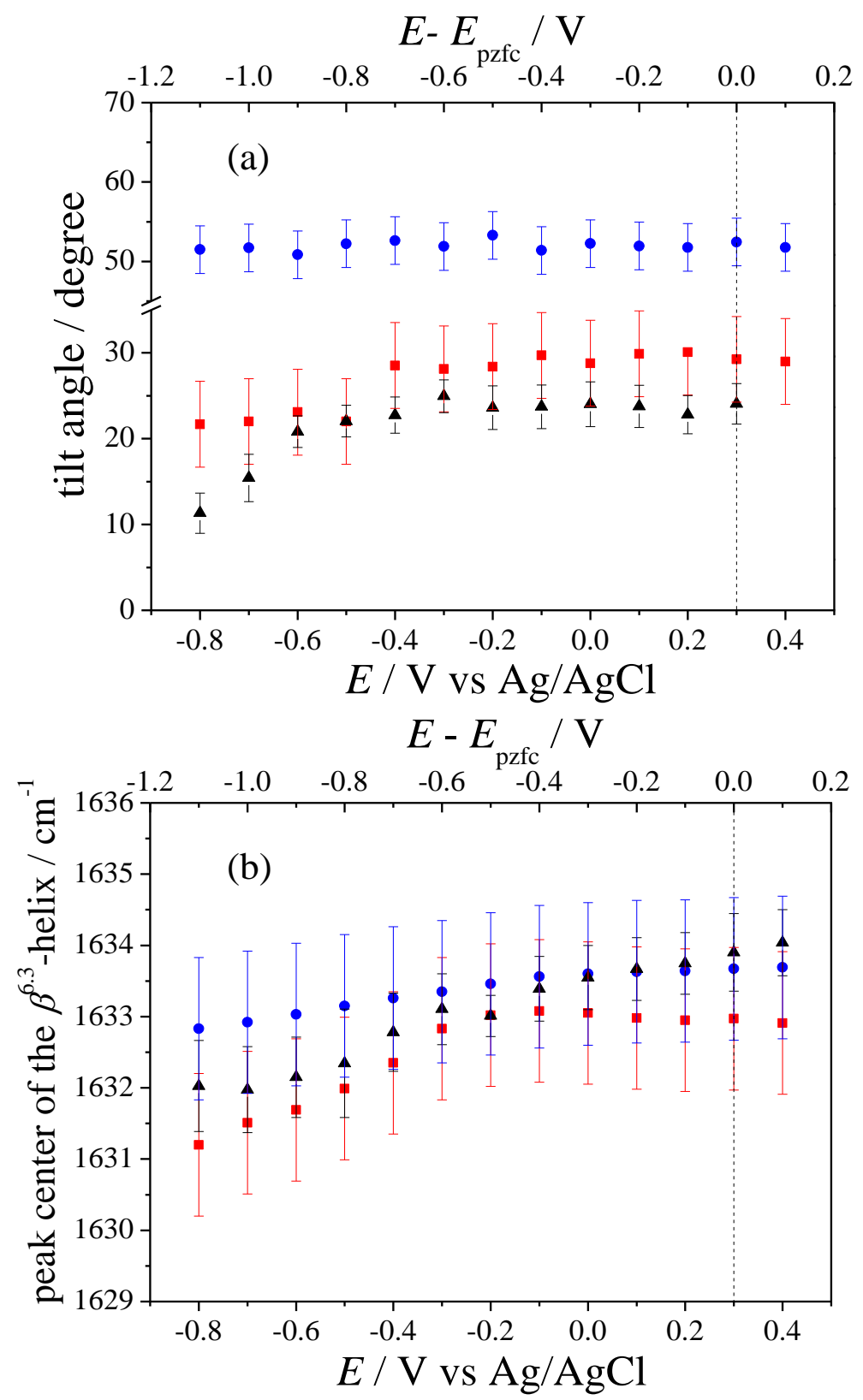

Figure 8. (a) Tilt angle and (b) frequency shift of the IR peak center of the $\beta^{6.3}$-helix in gA-DPhPC (10:90) / DPTL (red square) and gA-DPhPC (20:80) / DPTL (blue circle) in the tethered bilayers and the data from the gramicidin-DMPC(10:90) (black triangles) supported bilayer at gold (111) surface plotted as a function of the electrode potential. The data of the supported bilayer are taken from Ref. [26]. The upper horizontal axis plots potential drop across the bilayer. 
direct contact of the supported bilayer with the metal surface. However, the peak center frequency decreases further at potentials more negative than $-0.4 \mathrm{~V}$ indicating that the stress is released further when water penetrates into the space between the membrane and the metal. The peak center for the $\beta^{6.3}$-helix in the gA-DPhPC(20:80) / DPTL bilayer is shifted to higher wavenumbers indicating that at this high gramicidin concentration the molecules are not only randomly oriented but also that their $\beta^{6.3}$-helices are more stressed.

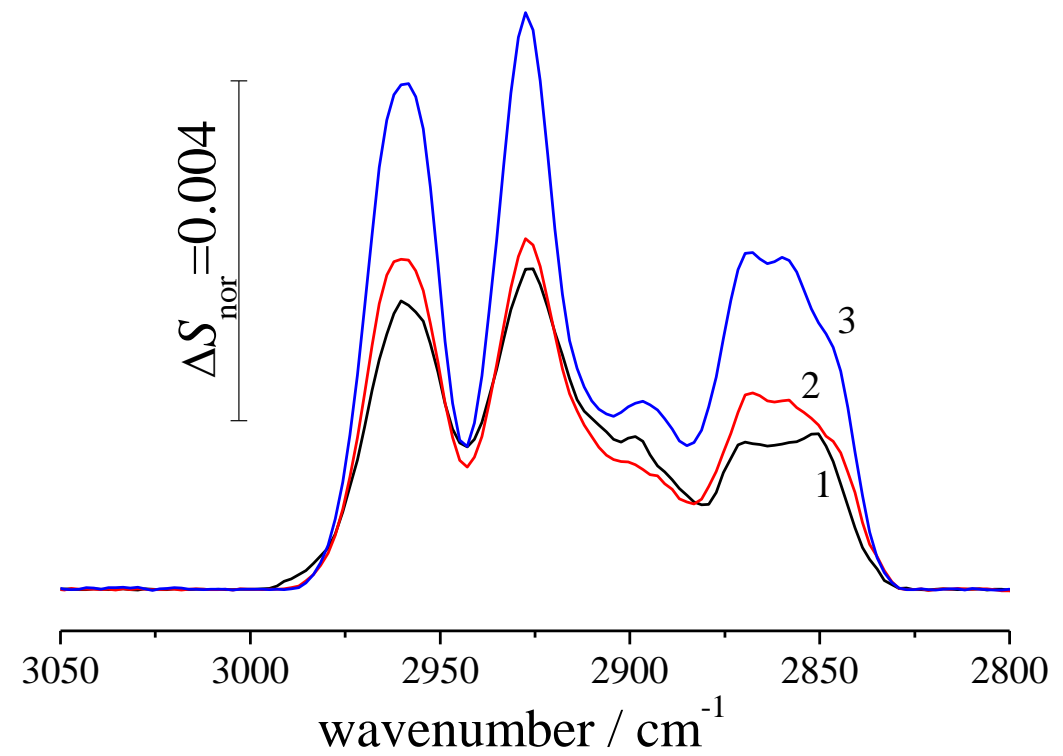

Figure 9. Normalized PM-IRRAS spectra of DPhPC/DPTL (1, black), gA-DPhPC(10:90) / DPTL (2, red) and gA-DPhPC(20:80) / DPTL (3, blue) tethered bilayers in the C-H stretching region; $0.1 \mathrm{M} \mathrm{KClO}_{4} / \mathrm{D}_{2} \mathrm{O}$ electrolyte, $E=0.0 \mathrm{~V}$ vs $\mathrm{Ag} / \mathrm{AgCl}$.

Figure 9 shows the normalized PM-IRRAS spectra in the $\mathrm{C}-\mathrm{H}$ stretching region of bilayers that are peptide-free and containing 10 and $20 \mathrm{~mol} \%$ of gA. These spectra consist of many overlapping bands, which can be deconvoluted using the procedure described by Leitch et al. [35]. The integrated intensities of the deconvoluted symmetric and asymmetric $\mathrm{CH}_{2}$ could be used to determine the average tilt angle of the diphytanoyl chains with the help of Eq. 4 and 5. The average tilt angle of the diphytanoyl chains ( $\left.\theta_{\text {chain }}\right)$ 
in the tBLM without gA is equal to $31 \pm 4^{\circ}$ and is comparable to the value $29 \pm 5^{\circ}$ of $\theta_{\text {chain }}$ in the monolayer of DPTL self-assembled on Au (111) surface [35]. In the presence of gramicidin, the $\mathrm{CH}_{2}$ group vibrations in diphytanoyl chains of the lipids overlap with vibrations in the side chains of gA molecules. However, the band of the methyl group in the middle of the phytanoyl chain $\left(\mathrm{CH}_{3}-\mathrm{m}\right)$ at $\sim 2868 \mathrm{~cm}^{-1}$ is free from spectral interferences of vibrations in gramicidin molecules. The transition dipole of the $\mathrm{CH}_{3}-\mathrm{m}$ band is normal with respect to the trans fragment of the phytanoyl chain [35]. Therefore, the integrated intensity of this band could be used to calculate the average tilt angle of the trans fragments of phytanoyl chains as shown in ref [26]. These calculations give values of $28 \pm 5^{\circ}, 40 \pm 4^{\circ}$ and $60 \pm 3^{\circ}$ for the average tilt of trans fragments of phytanoyl chains in bilayers with 0,10 and $20 \mathrm{~mol} \%$ of gA. These numbers change little with the applied potential and the changes are within experimental uncertainties.

For the bilayer without $\mathrm{gA}$, the tilt angle calculated from the $\mathrm{CH}_{3}-\mathrm{m}$ band falls within experimental error of the tilt angles calculated from the intensities of symmetric and asymmetric $\mathrm{CH}_{2}$ bands. For the bilayer with $20 \mathrm{~mol} \%$ of $\mathrm{gA}$ added to the top leaflet, the lipid molecules are either disordered or assume a nearly parallel orientation with respect to the electrode surface. For the bilayer with $10 \mathrm{~mol} \%$ of gA added to the top leaflet, the tilt of phytanoyl chains is about $10^{\circ}$ higher than the tilt of $\beta^{6.3}$-helices of gA molecules. Similar results were observed for gA molecules reconstituted into the DMPC bilayers supported at the $\mathrm{Au}(111)$ electrodes [26]. In order to form ion channels, the length of the $\beta^{6.3}$-helices projected onto the surface normal should match the thickness of the hydrophobic portion of the bilayer $[58,59]$. The thickness of the hydrophobic core in the DMPC bilayer at $30{ }^{\circ} \mathrm{C}$ (liquid crystalline state) is $\sim 2.7 \mathrm{~nm}$ [36]. Similar thickness can be expected for the hydrophobic core of the DPhPC/DPTL bilayer since the lipids are also in a liquid crystalline state. The length of the $\beta^{6.3}$-helix of $\mathrm{gA}$ is equal to $1.3 \mathrm{~nm}$ [60]. For the average tilt angle $29 \pm 4^{\circ}$, the length projected onto the surface normal is $1.14 \pm 0.05 \mathrm{~nm}$. When two gA molecules form the ion channel in the bilayer, the length of the channel 
projected onto the surface normal amounts to $2.3 \pm 0.1 \mathrm{~nm}$. This value is about $0.4 \mathrm{~nm}$ smaller than the thickness of the hydrophobic core for the peptide-free bilayer. This corresponds to the hydrophobic mismatch between the thickness of the hydrophobic core of the lipids and the projected length of the channel. To eliminate the mismatch, the lipids become more tilted to reduce the thickness of the hydrophobic core of the bilayer. The requirement of the hydrophobic matching explains the change in the tilt angle of the lipids in the bilayer without and with $10 \mathrm{~mol} \% \mathrm{gA}$.

In addition to the amide I stretching band at $1632 \mathrm{~cm}^{-1}$, the IR band intensities at wavenumbers higher than $1640 \mathrm{~cm}^{-1}$ are also presented in Figure 6(a). For a well ordered sample, changes in these band intensities depend on changes in the orientation and concentration, however, the bands at 1644, 1653,1660, 1669, $1678 \mathrm{~cm}^{-1}$ are assigned to the double helix (DH) conformation. As a consequence of the large number of vibrational overlap in this region and the fact that the direction of their transition dipoles is unknown, it is difficult to discuss changes of their intensity. For the carbonyl band, the concentration of carbonyl group decreases by moving from the bilayer with $10 \%$ to $20 \%$ of gA. However, the decrease in the concentration of $\mathrm{C}=\mathrm{O}$ groups is compensated by a more tilted orientation of the phytanyl chains of the lipid. The direction of the transition dipole of the carbonyl band is almost perpendicular to the chain direction. When chains tilt, it increases the component of the transition dipole in the direction normal to the surface increasing the band intensity.

\section{Summary and Conclusions}

The DPhPC/DPTL bilayer tethered to the gold (111) electrode surface was employed to investigate ion channel formation by gramicidin A molecules. In this architecture, the hydrophobic core of this membrane was separated from the metal by the tetraethylene glycol spacer. The EIS measurements demonstrated that gA is reconstituted into both leaflets of the bilayer and is capable of forming an active ion channels when 
concentration of $\mathrm{gA}$ in the membrane is less than or equal to $10 \mathrm{~mol} \%$. At higher $\mathrm{gA}$ concentrations, the conductivity of the membrane decreases. The PM-IRRAS measurements provided molecular level interpretation to the electrochemical data. The IR spectra demonstrated that the channel forming $\beta^{6.3}$-helix conformation of gA molecules is predominant in the bilayer with $10 \mathrm{~mol} \%$ of gA. The $\beta^{6.3}$-helices assume a small tilt angle of $29 \pm 4^{\circ}$ with respect to the surface normal, which is favorable for the formation of ion conducting dimers. In the presence of gramicidin peptides, the phytanoyl chains of the membrane adopt a larger tilt angle to adjust the thickness of its hydrophobic layer to the length of the peptide. The PM-IRRAS studies have also shown that the presence of the TEG spacer, which separates the hydrophobic portion of the membrane from the metal,

alleviates the stress in the $\beta^{6.3}$-helix of gA molecules. The PM-IRRAS measurements of the bilayer that contained $20 \mathrm{~mol} \%$ of the gramicidin showed that gA peptides assume a random orientation that prevents the ion channel dimer formation since the $\beta^{6.3}$-helices of gA are stressed. Additionally, the lipid chains are highly disordered in membranes with 20 mol\% peptide content. The results of this paper illustrate that a combination of EIS and PM-IRRAS techniques provide a unique opportunity to attain the molecular level interpretation to the conductivity measurements of ion channels reconstituted into a biomimetic membrane.

\section{Acknowledgements}

This work was supported by a grant from Natural Sciences and Engineering Research Council of Canada (NSERC). JL acknowledges a Canada Research Chair Award.

\section{Supplementary Materials Available:}

The optical constants of the gA-DPhPC mixture are shown in Figure S1. The 2D-COS and FSD results of the PM-IRRAS spectra of the gA-DPhPC(10:90) /DPTL tBLM are 
plotted in Figures S2 and S3, respectively. Figure S4 show the PM-IRRAS spectra of the gA-DPhPC (20:80) / DPTL tBLM at different electrode potentials.

\section{References:}

[1] E.K. Sinner, W. Knoll, Functional tethered membranes, Curr. Opin. Chem. Biol., 5 (2001) 705-711.

[2] S.M. Schiller, R. Naumann, K. Lovejoy, H. Kunz, W. Knoll, Archaea analogue thiolipids for tethered bilayer lipid membranes on ultrasmooth gold surfaces, Angew. Chem. Int. Ed., 42 (2003) 208-211.

[3] M. Frasconi, F. Mazzei, T. Ferri, Protein immobilization at gold-thiol surfaces and potential for biosensing, Anal. Bioana.l Chem., 398 (2010) 1545-1564.

[4] C. Rossi, J. Chopineau, Biomimetic tethered lipid membranes designed for membrane-protein interaction studies, Eur. Biophys. J., 36 (2007) 955-965.

[5] W. Knoll, I. Koeper, R. Naumann, E.-K. Sinner, Tethered bimolecular lipid membranes - A novel model membrane platform, Electrochimica Acta, 53 (2008) 6680-6689.

[6] R. Naumann, D. Walz, S.M. Schiller, W. Knoll, Kinetics of valinomycin-mediated K+ ion transport through tethered bilayer lipid membranes, J. Electrochem. Chem., 550 (2003) 241-252.

[7] L. Becucci, M. Innocenti, E. Salvietti, A. Rindi, I. Pasquini, M. Vassalli, M.L. Foresti, R. Guidelli, Potassium ion transport by gramicidin and valinomycin across a $\mathrm{Ag}(111)$-supported tethered bilayer lipid membrane, Electrochimica Acta, 53 (2008) 6372-6379.

[8] L.H. He, J.W.F. Robertson, J. Li, I. Karcher, S.M. Schiller, W. Knoll, R. Naumann, Tethered bilayer lipid membranes based on monolayers of thiolipids mixed with a complementary dilution molecule. 1. Incorporation of channel peptides, Langmuir, 21 (2005) 11666-11672.

[9] M.R. Moncelli, L. Becucci, S.M. Schiller, Tethered bilayer lipid membranes self-assembled on mercury electrodes, Bioelectrochemistry, 63 (2004) 161-167.

[10] L. Becucci, A. Santucci, R. Guidelli, Gramicidin conducting dimers in lipid bilayers are stabilized by single-file ionic flux along them, J. Phys. Chem. B, 111 (2007) 9814-9820.

[11] J. Kozuch, C. Steinem, P. Hildebrandt, D. Millo, Combined electrochemistry and surface-enhanced infrared absorption spectroscopy of gramicidin A incorporated into tethered bilayer lipid membranes, Angew. Chem. Int. Ed., 51 (2012) 8114-8117.

[12] L. Becucci, R. Guidelli, Can gramicidin ion channel affect the dipole potential of neighboring phospholipid headgroups?, Bioelectrochemistry, 106 (2015) 343-352.

[13] L. Becucci, F. Maran, R. Guidelli, Probing membrane permeabilization by the antibiotic lipopeptaibol trichogin GA IV in a tethered bilayer lipid membrane, Biochim. Biophys. Acta, 1818 (2012) 1656-1662.

[14] L. Becucci, R. Guidelli, Kinetics of channel formation in bilayer lipid membranes (BLMs) and tethered BLMs: Monazomycin and melittin, Langmuir, 23 (2007) 5601-5608.

[15] W.R. Veatch, E.T. Fossel, E.R. Blout, Conformation of gramicidin A, Biochemistry, 13 (1974) 
5249-5256.

[16] N. Abdulmanan, J.F. Hinton, Conformation States of Gramicidin A along the Pathway to of Channels in Model Membranes Determined by 2D NMR and Circular Dichroism Spectroscopy, Biochemistry, 33 (1994) 6773-6783.

[17] B.M. Burkhart, D.A. Langs, W.A. Pangborn, W.L. Duax, V. Pletnev, R.M. Gassman, Gramicidin D conformation, dynamics and membrane ion transport, Biopolymers, 51 (1999) 129-144.

[18] S. Weinstein, B.A. Wallace, E.R. Blout, J.S. Morrow, W. Veatch, Conformation of gramicidin A channel in phospholipid vesicles: A carbon-13 and fluorine-19 nuclear magnetic resonance study, Proc. Nat.l Acad. Sci. USA, 76 (1979) 4230-4234.

[19] B.A. Wallace, Gramicidin channels and pores, Annu. Rev. Biophys. Biophys., 19 (1990) $127-157$.

[20] D.A. Langs, G.D. Smith, C. Courseille, G. Precigoux, M. Hospital, Monoclinic uncomplexed double-stranded, antiparallel, left-handed beta 5.6-helix (increases decreases beta 5.6) structure of gramicidin A: alternate patterns of helical association and deformation, Proc. Nat.l Acad. Sci. USA, 88 (1991) 5345-5349.

[21] C. Steinem, A. Janshoff, H.J. Galla, M. Sieber, Impedance analysis of ion transport through gramicidin channels incorporated in solid supported lipid bilayers, Bioelectrochem Bioenerg, 42 (1997) 213-220.

[22] M. Naumowicz, Z. Figaszewski, Impedance analysis of phosphatidylcholine membranes modified with gramicidin D, Bioelectrochemistry, 61 (2003) 21-27.

[23] A.H. Kycia, Z. Su, C.L. Brosseau, J. Lipkowski, In Situ PM-IRRAS Studies of Biomimetic Membranes Supported at Gold Electrode Surfaces, in: A. Wieckowski, C. Korzeniewski, B. Braunschweig (Eds.) Vibrational Spectroscopy at Electrified Interfaces, Wiley-VCH, Weinheim, 2013, pp. 345-417.

[24] A. Barth, Infrared spectroscopy of proteins, Biochim. Biophys. Acta, 1767 (2007) 1073-1101.

[25] J. Ha, C.S. Henry, I. Fritsch, Formation and characterization of supported hexadecanethiol dimyristoyl phosphatidylcholine hybrid bilayers containing gramicidin D, Langmuir, 14 (1998) 5850-5857.

[26] T. Laredo, J.R. Dutcher, J. Lipkowski, Electric field driven changes of a gramicidin containing lipid bilayer supported on a Au(111) surface, Langmuir, 27 (2011) 10072-10087.

[27] J. Kunze, J. Leitch, A.L. Schwan, R.J. Faragher, R. Naumann, S. Schiller, W. Knoll, J.R. Dutcher, J. Lipkowski, New method to measure packing densities of self-assembled thiolipid monolayers, Langmuir, 22 (2006) 5509-5519.

[28] Y. Barenholz, D. Gibbes, B.J. Litman, J. Goll, T.E. Thompson, F.D. Carlson, Simple method for preparation of homogeneous phospholipid vesicles, Biochemistry, 16 (1977) 2806-2810.

[29] N.H. Li, V. Zamlynny, J. Lipkowski, F. Henglein, B. Pettinger, In situ IR reflectance absorption spectroscopy studies of pyridine adsorption at the $\mathrm{Au}(110)$ electrode surface, J. Electrochem. Chem., 524 (2002) 43-53.

[30] V. Zamlynny, J. Lipkowski, Quantitative SNIFTIRS and PM IRRAS of organic molecules at electrode surfaces, in: R. Alkire, D.M. Kolb, J. Lipkowski (Eds.) Advances in Electrochemical Science and Engineering: Diffraction and Spectroscopic Methods in Electrochemistry, Wiley-VCH, Weinheim, 2006, pp. 315-376.

[31] V. Zamlynny, PhD thesis, University of Guelph (Canada), 2002. 
[32] E. Palik, Handbook of Optical Constants of Solids II, Academic Press, San Diego, 1998.

[33] J.E. Bertie, M.K. Ahmed, H.H. Eysel, Infrared intensities of liquids. 5. Optical and dielectric constants, integrated intensities, and dipole moment derivatives of $\mathrm{H} 2 \mathrm{O}$ and $\mathrm{D} 2 \mathrm{O}$ at 22 degree, J. Phys. Chem., 93 (1989) 2210-2218.

[34] B.D.L. R.J. Lipert, M.D. Porter, Specular reflection spectroscopy, John Wiley \& Sons, Inc., New York, 1998.

[35] J. Leitch, J. Kunze, J.D. Goddard, A.L. Schwan, R.J. Faragher, R. Naumann, W. Knoll, J.R. Dutcher, J. Lipkowski, In situ PM-IRRAS studies of an archaea analogue thiolipid assembled on a au(111) electrode surface, Langmuir, 25 (2009) 10354-10363.

[36] J.F. Nagle, S. Tristram-Nagle, Structure of lipid bilayers, Biochim. Biophys. Acta, 1469 (2000) $159-195$

[37] D. Allara, J. Swalen, An infrared reflection spectroscopy study of oriented cadmium arachidate monolayer films on evaporated silver, J. Phys. Chem., 86 (1982) 2700-2704.

[38] D.L. Allara, R.G. Nuzzo, Spontaneously organized molecular assemblies. 2. Quantitative infrared spectroscopic determination of equilibrium structures of solution-adsorbed n-alkanoic acids on an oxidized aluminum surface, Langmuir, 1 (1985) 52-66.

[39] J. Umemura, T. Kamata, T. Kawai, T. Takenaka, Quantitative evaluation of molecular orientation in thin Langmuir-Blodgett films by FT-IR transmission and reflection-absorption spectroscopy, J. Phys. Chem., 94 (1990) 62-67.

[40] J.J. Leitch, C.L. Brosseau, S.G. Roscoe, K. Bessonov, J.R. Dutcher, J. Lipkowski, Electrochemical and PM-IRRAS characterization of cholera toxin binding at a model biological membrane, Langmuir, 29 (2013) 965-976.

[41] L. Tamm, S.A. Tatulian, Infrared spectroscopy of proteins and peptides in lipid bilayers, Q. Rev. Biophys., 30 (1997) 365-429.

[42] D. Marsh, M. Muller, F.-J. Schmitt, Orientation of the infrared transition moments for an $\alpha$-helix, Biophys. J., 78 (2000) 2449-2510.

[43] Z. Kota, T. Pali, D. Marsh, Orientation and lipid-peptide interactions of gramicidin A in lipid membranes: Polarized attenuated total reflection infrared spectroscopy and spin-label electron spin resonance, Biophys. J., 86 (2004) 1521-1531.

[44] V.M. Naik, S. Krimm, Vibrational analysis of the structure of gramicidin A. I. Normal mode analysis, Biophys. J., 49 (1986) 1131-1145.

[45] W.P. Ulrich, H. Vogel, Polarization-modulated FTIR spectroscopy of lipid/gramicidin monolayers at the water interface, Biophys. J., 76 (1999) 1639-1647.

[46] E. Nabedryk, M.P. Gingold, J. Breton, Orientation of gramicidin A transmembrane channel. Infrared dichroismstudy of gramicidin in vesicles, Biophys. J., 38 (1982) 243-249.

[47] U.W. Hamm, D. Kramer, R.S. Zhai, D.M. Kolb, The pzc of Au(111) and Pt(111) in a perchloric acid solution: An ex situ approach to the immersion technique, J. Electrochem. Chem., 414 (1996) 85-89.

[48] P. Ramírez, R. Andreu, Á. Cuesta, C.J. Calzado, J.J. Calvente, Determination of the potential of zero charge of Au (111) modified with thiol monolayers, Anal. Chem., 79 (2007) 6473-6479.

[49] Z.F. Su, J. Leitch, J. Lipkowski, Measurements of the Potentials of Zero Free Charge and Zero Total Charge for 1-thio-beta-D-glucose and DPTL Modified Au(111) Surface in Different Electrolyte 
Solutions, Z. Phys. Chem., 226 (2012) 995-1009.

[50] R. Seenath, J.J. Leitch, Z. Su, R.J. Faragher, A.L. Schwan, J. Lipkowski, Measurements of surface concentration and charge number per adsorbed molecule for a thiolipid monolayer tethered to the $\mathrm{Au}$ (111) surface by long a hydrophilic chain, J. Electrochem. Chem., (2016).

[51] S. Sek, T. Laredo, J.R. Dutcher, J. Lipkowski, Molecular Resolution Imaging of an Antibiotic Peptide in a Lipid Matrix, J. Am. Chem. Soc., 131 (2009) 6439-6444.

[52] L. Becucci, V. Tramonti, A. Fiore, V. Fogliano, A. Scaloni, R. Guidelli, Channel-forming activity of syringomycin $\mathrm{E}$ in two mercury-supported biomimetic membranes, Biochimica et Biophysica Acta (BBA)-Biomembranes, 1848 (2015) 932-941.

[53] L. Becucci, G. Aloisi, R. Guidelli, When and how the melittin ion channel exhibits ohmic behavior, Bioelectrochemistry, 113 (2017) 51-59.

[54] R. Guidelli, L. Becucci, Ion transport across biomembranes and model membranes, J. Solid State Electrochem., 15 (2011) 1459-1470.

[55] Y.O. I. Noda, wo-dimensional correlation spectroscopy: applications in vibrational and optical spectroscopy, John Wiley \&Sons, Great Britain, 1994.

[56] W.K. Surewicz, H.H. Mantsch, New insight into protein secondary structure from resolution-enhanced infrared spectra, Biochim. Biophys. Acta, 952 (1988) 115-130.

[57] A. Barth, C. Zscherp, What vibrations tell about proteins, Q. Rev. Biophys., 35 (2002) 369-430.

[58] T.A. Harroun, W.T. Heller, T.M. Weiss, L. Yang, H.W. Huang, Experimental evidence for hydrophobic matching and membrane-mediated interactions in lipid bilayers containing gramicidin, Biophys. J., 76 (1999) 937-945.

[59] T.A. Harroun, W.T. Heller, T.M. Weiss, L. Yang, H.W. Huang, Theoretical analysis of hydrophobic matching and membrane-mediated interactions in lipid bilayers containing gramicidin, Biophys. J., 76 (1999) 3176-3185.

[60] R.E. Koeppe, J.M. Berg, K.O. Hodgson, L. Stryer, Gramicidin A crystals contain two cation binding sites per channel, Nature, 279 (1979) 723-725. 